\title{
Lactulose: a model system to investigate solid state amorphization induced by milling
}

\author{
F. Ngono ${ }^{1,2}$, J.F. Willart ${ }^{1, *}$, G. J. Cuello², M. Jimenez-Ruiz ${ }^{2}$, F. Affouard ${ }^{1}$ \\ ${ }^{1}$ Université Lille Nord de France, Unité Matériaux et Transformations, UMR CNRS 8207, Av. Paul \\ Langevin, F-59655 Villeneuve d'Ascq, France \\ ${ }^{2}$ Institut Laue Langevin, 71 Av. des Martyrs, CS 20156, F-38042, Grenoble, France
}

\begin{abstract}
In this paper, we show that crystalline lactulose can be amorphized directly in the solid state by mechanical milling. Moreover, compared to similar materials, the amorphization kinetics of lactulose is found to be very rapid and the amorphous state thus obtained appears to be very stable against recrystallization upon heating. These features make lactulose a model compound for this type of solid state transformation. The ease of crystalline lactulose to be amorphized upon milling is explained by comparing elastic constants of lactulose with those of several other disaccharides. These constants have been determined by molecular dynamics simulations. The paper also shows how isothermal dissolution calorimetry can be used effectively for the determination of amorphization kinetics during grinding when the usual characterization techniques (DSC and powder X-ray diffraction) fail.
\end{abstract}

* Corresponding author at: Université Lille Nord de France, F-59655 Villeneuve d'Ascq, France.

E-mail address: Jean-Francois.Willart@univ-lille1.fr (J.F. Willart). 


\section{Introduction}

High energy mechanical milling is now recognized as an efficient technique to amorphize many crystalline pharmaceutical compounds ${ }^{1-4}$. This physical transformation results from a competition between an amorphization process induced by the mechanical shocks and a subsequent thermally activated recrystallization of the mechanically amorphized fractions ${ }^{5-7}$. A total amorphization thus requires that the material be milled at temperatures well enough below its glass transition temperature to prevent any recrystallization ${ }^{8-10}$.

This amorphization route has the advantage over the usual quench of the liquid to operate directly in the solid state ${ }^{1,11}$. It thus avoids any melting stage which often induces a more or less strong degradation of molecular materials ${ }^{12}$. The amorphization upon milling has also the advantage over spray-drying and freeze-drying ${ }^{13}$ amorphization techniques to avoid any dissolution stage in a solvent. Such a dissolution sometimes induces conformational changes of molecules modifying the nature of the amorphous state ${ }^{14}$. Moreover, some remaining traces of solvents after drying have both a toxic character and a plasticizing effect which can decrease the physical stability of the amorphous state.

On the other hand, milling has the disadvantage of generally requiring very long milling times 15,16 (typically several hours in a high energy planetary mill) and sometimes at very low temperatures ${ }^{17,18}$ (cryomilling). In addition, the amorphous state obtained upon milling is generally less stable than those obtained by melt quenching and dissolution techniques. Several explanations have already been put forward to explain this quasi systematic lower stability of milling induced amorphous materials ${ }^{19}$ : (i) Some remaining small crystallites could have escaped the milling and act as nuclei which promote the recrystallization, (ii) the short range order characterizing the amorphous state can be slightly increased due to a reminiscence of the initial crystalline order. This can potentially modify the nucleation and growth rates which drive the recrystallization propensity of the material. (iii) The high specific surface of the powder after milling ${ }^{20}$ can promote surface crystallization which has been shown to be much faster than bulk crystallization ${ }^{21-24}$.

In this paper, we investigate the propensity of lactulose $\left(\mathrm{C}_{12} \mathrm{H}_{22} \mathrm{O}_{11}\right)$ to be amorphized by mechanical milling. Special attention will be paid to the physical stability of the amorphized fractions and to the rate of the amorphization process. The latter will be determined by an original technique of isothermal dissolution calorimetry whose advantages over conventional techniques (PXRD and DSC) will be clearly demonstrated. The rate of the amorphization process will be compared to that of other disaccharides and the differences will be discussed according to the elastic constants of these sugars. These elastic constants, which are lacking in the literature, have been determined here by molecular dynamic simulations.

It must be noted that lactulose is characterized by a rich tautomerism so that the tautomeric composition of its amorphous state can be modified by the amorphization technique itself. These tautomeric changes have been studied in details and have been found to have no influence on the glass transition properties of amorphous lactulose studied in this paper. However, the effects of tautomerism have been found to be strong in the case of amorphous 
lactulose produced by spray-drying and freeze-drying. All these effects due to mutarotation will be presented in detail in a forthcoming paper. 


\section{Experimental and simulation sections 2.1 Materials}

Crystalline anhydrous lactulose was purchased from Sigma Aldrich. Thermogravimetric experiments have shown that it contains about $3.5 \%$ of water inclusions. The commercial form was thus slightly crushed to favour water release, and dried at $70{ }^{\circ} \mathrm{C}$ during $15 \mathrm{~min}$. The obtained sample has been checked to be totally crystalline, and has been used for the different experiments.

\subsection{Ball milling}

The crystalline material was milled at room temperature (RT) using a high energy planetary mill (Pulverisette 7-Fritsch, Idar-Oberstein, Germany). We used $\mathrm{ZrO}_{2}$ milling jars of $43 \mathrm{~cm}^{3}$ with seven balls $(\phi=15 \mathrm{~mm}$ ) of the same material. One gram of material was placed in the planetary mill corresponding to a ball-sample weight ratio of 75:1. The rotation speed of the solar disk was set to $400 \mathrm{rpm}$ that corresponds to an average acceleration of the milling balls of $5 \mathrm{~g}$. We took care to alternate milling periods (typically $15 \mathrm{~min}$ ) with pause periods (typically $15 \mathrm{~min}$ ) to limit the mechanical heating of the sample.

\subsection{Thermogravimetric Analysis (TGA)}

The thermogravimetric analyses were performed with the TGA Q500 of TA Instruments (Guyancourt, France). During the experiment, the sample was placed in an open platinum sample pan and flushed with a highly pure nitrogen gas. The temperature reading was calibrated using the Curie points of alumel and nickel, while the mass reading was calibrated using the balance tare weights provided by TA Instruments.

\subsection{Differential Scanning Calorimetry (DSC) experiments}

The differential scanning calorimetry (DSC) experiments were performed with a Q1000 calorimeter of TA Instruments (Guyancourt, France). For all the measurements, the calorimeter head was flushed with highly pure nitrogen gas. Temperature and enthalpy readings were calibrated using pure indium at the same scan rates used in the experiments. The samples have been placed in open pans (pans with no lid) to allow any water absorbed during the milling process to evaporate upon heating. For each scan, a small sample of approximately $4 \mathrm{mg}$ of lactulose was used to achieve good resolution and good thermal conductivity. Some experiments were performed in the temperature modulated mode (MDSC) using a heating rate of $5{ }^{\circ} \mathrm{C} / \mathrm{min}$, a modulation amplitude of $0.663{ }^{\circ} \mathrm{C}$ and a modulation period of $50 \mathrm{~s}$. Those parameters correspond to "heat only" conditions.

\subsection{Isothermal dissolution calorimetry experiments}

Dissolution enthalpy of the different samples was measured in the C80 calorimeter of Setaram (France) using reversal mixing cells in hasteloy. The dissolutions were performed at $37{ }^{\circ} \mathrm{C}$ in water. This water was purified using the AQUADEM system of VEOLIA, where the resulting water has a conductivity higher than $180 \mathrm{M} \Omega$. For each experiment, $100 \mathrm{mg}$ of sample (solute) 
was systematically dissolved in $3.80 \mathrm{~g}$ of water (solvent). Since the water solubility of lactulose is $764 \mathrm{~g} / \mathrm{L}$ at $30{ }^{\circ} \mathrm{C}^{25}$, the dilution factor is close to 30 which ensures total and fast dissolutions. A small amount of paraffin oil was used to improve the tightness of the solvent compartment before the dissolution stage. This oil has been checked to have no effect on the dissolution enthalpy.

\subsection{X-ray diffraction experiments}

The powder X-ray diffraction (XRD) experiments were performed with a PanAlytical X'PERT PRO MPD (Almelo, The Netherlands) diffractometer $\left(\lambda_{\mathrm{CuK} \alpha}=1.5418 \AA\right.$ for combined $\mathrm{K}_{\alpha 1}$ and $\mathrm{K}_{\alpha 2}$ ) equipped with an $\mathrm{X}^{\prime}$ celerator detector (Almelo, The Netherlands). Samples were placed into Lindemann glass capillaries $(\phi=0.7 \mathrm{~mm})$. Data were recorded from $5^{\circ}$ to $40^{\circ} 2 \theta$ by step of $0.0167^{\circ} 2 \theta$ using a counting time of $50 \mathrm{~s}$ per step.

\subsection{Simulation details}

Molecular dynamics simulations have been performed using the DLPOLY package ${ }^{26}$ and the OPLS-AA force field ${ }^{27}$. This force field was chosen due to its capability of reproducing successfully a large number of experimental data for molecules with low molecular weight ${ }^{27,28}$. Simulations have been conducted either in the NPT or the NVT statistical ensemble where N is the number of molecules, $\mathrm{P}$ the pressure, $\mathrm{T}$ the temperature, and $\mathrm{V}$ the volume. The number of molecules, $\mathrm{N}$, was fixed in the simulations, and pressure and temperature were controlled with a Hoover barostat and thermostat, respectively. All NPT simulations were realized at zero pressure. A time step of 0.001 ps was chosen to integrate Newton's equation of motion. A cutoff radius of $10 \AA$ has been used to calculate short-range van der Waals interactions. Ewald summation was employed in order to calculate the long-range electrostatic interactions with the same cutoff radius. Periodic boundary conditions were applied in all directions.

Elastic constants of lactulose, sucrose, trehalose and lactose were estimated using the explicit deformation method ${ }^{29}$. For each disaccharide, a crystalline simulation box has been generated from the experimental crystallographic data $\left(\right.$ lactulose ${ }^{30}$, sucrose $^{31}$, trehalose $^{32}$ and lactose $\mathrm{e}^{33}$ ), with $\mathrm{N}=336,280,384$ and 280 molecules, respectively. Each system was first equilibrated at $\mathrm{T}=50 \mathrm{~K}$ for $600 \mathrm{ps}$ in the statistical ensemble NPT and then for $300 \mathrm{ps}$ in the NVT ensemble using the equilibrated volume obtained from the NPT simulation. This low temperature has been chosen in order to be certain to remain in the elastic regime. Then, different deformation types were applied to each box in the NVT ensemble. After short equilibration, a simulation of about $300 \mathrm{ps}$ is run to obtain the equilibrium stress tensor $\sigma$ under a given deformation $\varepsilon$. The full set of elastic constants can then be retrieved from several deformations using the generalised Hooke's law $\sigma_{\mathrm{i}}=\mathrm{C}_{\mathrm{ij}} \varepsilon_{\mathrm{j}}$, using implicit sum convention with $\mathrm{j}=1$ to 6 and the Voigt notation $(1=\mathrm{xx}, 2=\mathrm{yy}, 3=\mathrm{zz}, 4=\mathrm{yz}, 5=\mathrm{xz}$ and $6=\mathrm{xy})$. From the full set of elastic constants $\mathrm{C}_{\mathrm{ij}}$, the bulk modulus $\mathrm{B}_{\mathrm{v}}$ and shear modulus $\mathrm{G}_{\mathrm{v}}$ are calculated according to the Voigt approximation (see Eq. 2 in Zhou et al. ${ }^{34}$ ). 


\section{Results and discussion}

\subsection{Melt quenching of lactulose}

Figure 1 presents the XRD pattern of the commercial lactulose (black line). It shows Bragg peaks characteristic of a crystalline state. The analysis of this XRD pattern with the FullProf software ${ }^{35}$ indicates that the system is monoclinic, with cell parameters compatible with those obtained by Jeffrey et al. for anhydrous crystalline lactulose ${ }^{30}$.

Figure 2 shows DSC curves of lactulose. Run 1 corresponds to the heating $\left(5^{\circ} \mathrm{C} / \mathrm{min}\right)$ of the crystalline material. It only shows a melting endotherm which confirms the crystalline character of the material. The temperature and enthalpy of melting are respectively $\mathrm{T}_{\mathrm{m}}=162 \pm 1^{\circ} \mathrm{C}$ and $\Delta \mathrm{H}_{\mathrm{m}}=125 \pm 1 \mathrm{~J} / \mathrm{g}$. Run 2 corresponds to the cooling $\left(5^{\circ} \mathrm{C} / \mathrm{min}\right)$ of the melt down to $20^{\circ} \mathrm{C}$. No sign of exothermic crystallization can be detected, which means that the liquid can be easily undercooled using a moderate cooling rate. The XRD pattern of the quenched melt (QM, green line) is also reported in figure 1. The absence of Bragg peaks in this XRD pattern confirms its amorphous character. This XRD pattern shows a main diffusion halo centred on the XRD pattern region of the crystal (black line) where the Bragg peaks are the most intense. Run 3 corresponds to the re-heating $\left(5^{\circ} \mathrm{C} / \mathrm{min}\right)$ of the quenched liquid. It clearly shows a specific heat jump $\left(\Delta \mathrm{C} p=0.64 \mathrm{~J} /\left(\mathrm{g} .{ }^{\circ} \mathrm{C}\right)\right)$ which indicates that a glassy state has been reached upon cooling the melt. The glass transition is located at $\mathrm{Tg}=94.7{ }^{\circ} \mathrm{C}$ which is in agreement with the value previously reported by Miller et al. ${ }^{36}$. Above $\mathrm{T} g$, no calorimetric accident is observed. In particular, no recrystallization exotherm is detected. This indicates that lactulose is a good glass former, which is reluctant to recrystallization upon heating at $5{ }^{\circ} \mathrm{C} / \mathrm{min}$.

\subsection{Effect of milling on crystalline lactulose}

Crystalline lactulose was milled in a planetary mill during $8 \mathrm{~h}$. The XRD pattern of the milled sample is reported in figure 1 (red line) where it can be compared to the non-milled crystal (black line). It appears that the Bragg peaks present in the non-milled material have totally disappeared after milling. The XRD pattern of the milled material only shows a large and diffuse halo characteristic of amorphous materials and which is fully similar to that of the quenched melt (green line). These results strongly suggest that lactulose has undergone a complete solid state amorphization upon milling.

Figure 3a shows TGA scan of lactulose milled $8 \mathrm{~h}$ recorded upon heating $\left(5^{\circ} \mathrm{C} / \mathrm{min}\right)$. The sample was previously dried at $60{ }^{\circ} \mathrm{C}$ during $10 \mathrm{~min}$. The figure shows one mass loss starting slowly around $130{ }^{\circ} \mathrm{C}$ and corresponding to the thermal degradation of the material.

Figure $3 \mathrm{~b}$ shows the total and reversible heat flows recorded during a MDSC heating scan (5 ${ }^{\circ} \mathrm{C} / \mathrm{min}$ ) of the $8 \mathrm{~h}$ milled lactulose (run 1). The DSC scan of the quenched melt (run 2) is also reported for comparison. The reversible signal shows two successive specific heat $(\mathrm{C} p)$ jumps. The first one, located at $88.6^{\circ} \mathrm{C}$, corresponds to the glass transition of the sample. This $\mathrm{Cp}$ jump proves that the crystal has been amorphized during the $8 \mathrm{~h}$ milling, and that the amorphous 
compound obtained is a glass. The second jump, located just after the first one, is an artefact which follows frequently the glass transition of finely divided amorphous materials. It is due to the microstructural change of the sample which passes from a fine powder to a dense liquid droplet just above Tg. This droplet has a much better apparent thermal conductivity than the fine powder resulting in an apparent increase of $\mathrm{Cp}$. Detailed information on this effect can be found in reference ${ }^{37}$. The total heat flow appears to be more complex. It shows the $\mathrm{Cp}$ jump associated with the glass transition previously detected in the reversible signal. This glass transition $\left(\mathrm{Tg}=85.7{ }^{\circ} \mathrm{C}\right)$ occurs at a slightly lower temperature than that detected in the reversible signal as the two signals probe the sample with different time scales ${ }^{38}$. It must also be noted that the glass transition of the milled material (red solid line) is preceded by a broad exotherm ranging from $74{ }^{\circ} \mathrm{C}$ to the onset of the glass transition. This event has a non-reversible character as it does not appear in the reversible signal. Such a sub-Tg exotherm is generally characteristic of hyper-quenched glasses ${ }^{39,40}$ and it has already been detected in several glasses obtained by milling ${ }^{41}$. It indicates that the amorphous lactulose obtained upon milling has reached a high enthalpy level similar to that of hyper-quenched glasses. At the end of the $\mathrm{Cp}$ jump, there is an endotherm related to the recovery of the enthalpy lost by the sample during its excursion below $\mathrm{Tg}$. This endotherm is followed by a broad endothermic signal starting slowly around $125^{\circ} \mathrm{C}$. It corresponds to the thermal degradation detected in the derivative TGA scan of figure $3 \mathrm{a}$.

Interestingly, the DSC scan of the $8 \mathrm{~h}$ milled lactulose (red solid line) shows no signs of recrystallization. The amorphous compound obtained by milling is therefore stable upon heating at $5{ }^{\circ} \mathrm{C} / \mathrm{min}$. This stability is quite exceptional, because amorphous compounds obtained by milling the crystal almost always recrystallize during heating at $5{ }^{\circ} \mathrm{C} / \mathrm{min}^{3,4,7,15}$.

The Tg of the quenched melt (run 2) occurs $8{ }^{\circ} \mathrm{C}$ above that of the milled material (run 1). This higher $\mathrm{Tg}$ is likely to be due to an anti-plasticization of the liquid by the degraded products generated at high temperature during the melting stage. This point is demonstrated in the next section.

\subsection{Effect of thermal degradation on the $\mathrm{Tg}$}

In order to study the influence of thermal degradation on the glass transition temperature, an amorphous lactulose sample obtained by milling was repeatedly scanned $\left(5^{\circ} \mathrm{C} / \mathrm{min}\right)$ by DSC for increasing final temperature. For each new scan, the final temperature is increased by $10^{\circ} \mathrm{C}$ compared to the previous one. Figure $4 \mathrm{~b}$ shows a schematic illustration of this thermal treatment, and figure $4 \mathrm{a}$ shows the DSC scans recorded for final temperature ranging from 125 ${ }^{\circ} \mathrm{C}$ to $185^{\circ} \mathrm{C}$.

The analysis of the DSC scans shown in figure 4a indicates that their evolution can be divided in two stages. The first stage corresponds to runs 1 and 2 . It shows no evolution of the glass transition temperature, whose value remains around $(85 \pm 2)^{\circ} \mathrm{C}$. The second stage corresponds to the last five DSC scans (runs 3 to 7). During this stage, the glass transition shifts by $20{ }^{\circ} \mathrm{C}$ toward the high temperatures as the maximum temperature reached by the sample varies from $145^{\circ} \mathrm{C}$ to $175^{\circ} \mathrm{C}$. Moreover, this evolution is accompanied by a strong spreading of the $\mathrm{Cp}$ jump and a slight decrease of its amplitude. It can also be noted that the end of each scan is 
marked by the onset of an endothermic signal corresponding to the thermal degradation of lactulose, as previously presented. Therefore, it is this thermal degradation, increasing with the maximum temperature reached by the sample, which is responsible for the shift of the glass transition toward the high temperatures seen in runs 3 to 7 . Thermal degradation of lactulose compounds thus appears to have a strong anti-plasticizing effect. This anti-plasticizing effect explains the higher glass transition temperature of the quenched melt in regard to that of the milled material seen in figure 3b. Milling thus appears to be a useful tool to amorphize safely materials which degrade upon melting.

In the course of run 1 (figure 4), the non-degraded amorphous lactulose obtained by milling was heated at $125^{\circ} \mathrm{C}$, i.e. just above Tg. At this temperature, the sample is in a metastable liquid state which does not recrystallize and does not degrade. After quenching, the physical state of the sample is thus equivalent to that of a non-degraded quenched liquid. The subsequent run 2 indicates that the glass transition temperature of this quenched liquid lactulose is close to that of the milled material $\left(\mathrm{T} g=85^{\circ} \mathrm{C}\right)$ which thus appears to be the true glass transition temperature of this material. The combination of milling and thermal treatments gives thus here the opportunity to produce a non-degraded liquid glass which cannot be safely obtained by the usual melt quenching of the crystal.

\subsection{Amorphization kinetics of crystalline lactulose under milling}

a- X-ray diffraction investigations

Figure 5 shows the XRD patterns of crystalline lactulose, recorded after different milling times varying from 0 to $8 \mathrm{~h}$. Each milling experiment has been performed with a fresh crystalline sample, and the XRD experiment has been performed immediately after the end of the milling process. All patterns were recorded at room temperature. They show a decrease of the intensity of Bragg peaks as well as the development of an underlying diffusion halo for increasing milling times. These effects are already perceptible after $5 \mathrm{~min}$ of milling, and their evolutions are fast so that the material is already almost totally X-ray amorphous after $1 \mathrm{~h}$ of milling. No further evolutions of the XRD pattern can be observed for longer milling times. This reflects the progressive amorphization of the material during milling. The evolution is also marked by a broadening of the Bragg peaks. This broadening reflects a reduction of the size of lactulose crystallites not yet amorphized, as well as the appearance of defects and micro strains.

\section{b- DSC investigations}

Figure 6 shows DSC curves (total heat flows) of crystalline lactulose recorded after different milling times ranging from 0 to $8 \mathrm{~h}$. Each milling experiment has been performed with a fresh crystalline sample, and the DSC experiment has been performed immediately after the end of the milling process. For all those experiments, the sample was initially dried at $60^{\circ} \mathrm{C}$ during 5 min to remove any residual water. The DSC scans show three main enthalpic events: 
- A specific heat $(\mathbf{C} p)$ jump at $\mathrm{T} g=85.5^{\circ} \mathrm{C}$. This jump is already detectable after $5 \mathrm{~min}$ of milling (it is more clearly seen on the dashed line, corresponding to the heat flow signal multiplied by 3 ). The amplitude of this jump increases progressively up to $1 \mathrm{~h}$ of milling where it is maximum. This indicates that a total crystal-to-glass conversion under milling occurs in about $1 \mathrm{~h}$. Beyond $1 \mathrm{~h}$ of milling, the amplitude of the jump remains unchanged.

- A crystallization exotherm already detectable after 5 min of milling. XRD experiments performed after this recrystallization showed that the milled material recrystallizes towards the initial crystalline form. This recrystallization occurs around $120{ }^{\circ} \mathrm{C}$ during the first 30 min of milling, and then shifts towards the high temperatures. This shift is frequently observed in compounds amorphizing under milling. This effect is generally attributed to a decrease in the crystalline fraction not yet amorphized which can serve as potential nuclei for the recrystallization of the amorphous fraction. It must also be noted that the shift is accompanied by a decrease of the recrystallization enthalpy, which even becomes zero after $8 \mathrm{~h}$ of milling. This indicates that an increasing fraction of the amorphous phase generated during milling no longer recrystallizes upon heating.

- A melting endotherm which overlaps more or less the crystallization exotherm. This melting endotherm presents a complex evolution with the milling time. Between 0 and 30 min of milling, the melting peak broadens and shifts toward the low temperatures. This behaviour is frequently observed in milled compounds. It is generally attributed to both the creation of defects in the crystallites and the strong crystallite size reduction ("Gibbs Thompson effect" 42 ) induced by milling. After 30 min of milling, the previous behaviour inverses and the melting endotherm shifts towards high temperatures. This indicates that the observed melting now concerns mostly crystallites coming from the recrystallization of the amorphous phase produced by the milling, rather than crystallites not yet amorphized as it was the case at the very beginning of the milling. The crystallites resulting from the recrystallization upon heating were not milled, and their melting is therefore not depressed by crystalline defects or size effects (i.e. a Gibbs Thomson effect). Furthermore, the melting enthalpy decreases and becomes zero after $8 \mathrm{~h}$ of milling. This behaviour is consistent with that of the recrystallization exotherm. It indicates that lactulose amorphizes totally upon milling and that an increasing part of the amorphized fraction loses its propensity to recrystallize upon heating at $5{ }^{\circ} \mathrm{C} / \mathrm{min}$ for increasing milling times.

c- Determination of the amorphization kinetic by isothermal dissolution calorimetry

The previous XRD and DSC results provide three potential means to determine the amorphization kinetic of lactulose upon milling, i.e. the evolution of the amorphous fraction $\mathrm{Xam}(\mathrm{t})$ as a function of the milling time $\mathrm{t}$. This requires to follow the time evolution of: 
(i) the decreasing Bragg peak intensities (figure 5). However, the experiments were performed here with different samples regularly taken from the milling jars. Due to the milling, these samples have strongly different grain size distributions giving rise to strongly different filling ratios of Lindeman capillaries and thus to inaccurate amorphous fraction determination.

(ii) the enthalpy of recrystallization. However, in the case of lactulose, the fraction amorphized during milling does not systematically recrystallize upon heating at 5 ${ }^{\circ} \mathrm{C} / \mathrm{min}$. This is particularly obvious for the sample milled $8 \mathrm{~h}$, which does not recrystallize at all whereas it is totally amorphous. This behaviour thus prevents the determination of amorphous fractions from the enthalpy of recrystallization.

(iii) the amplitude of the $\mathrm{Cp}$ jump at $\mathrm{Tg}$. However, in the present case, this measurement appears to be very inaccurate as the Cp jump merges with two other enthalpic events: the exotherm characteristic of hyperquenched glasses located just below $\mathrm{Tg}$ and the recrystallization exotherm located just above $\mathrm{Tg}$, especially for milling times shorter that $1 \mathrm{~h}$.

To get rid of the above difficulties, we have used isothermal dissolution calorimetry to determine the amorphization kinetic of lactulose upon milling. This technique allows an accurate determination of the dissolution enthalpy $\left(\Delta \mathrm{H}_{\text {dissol }}\right)$ of a material which generally differs strongly between its crystalline and amorphous forms ${ }^{36}$. After the establishment of a calibration curve using physical mixtures of totally crystalline and totally amorphous powders, an accurate determination of the amorphous contents of milled samples can be easily obtained. Dissolution calorimetry has the advantage over DSC to be a direct method to determine the amorphous content of a material. In particular, it does not require to heat the samples to induce a possible recrystallization of the amorphous fraction. Indeed, tiny structural changes in the milled material (e.g. disappearance of the very last nuclei or a subtle change in the local order) can have dramatic effects on the recrystallization upon heating while they only induce negligible changes in the energetic of the material. Dissolution calorimetry has also the advantage over XRD to be totally independent of the microstructure (e.g. grain size distribution) of the sample.

Figure 7 shows the heat flow curve recorded during the isothermal dissolution of crystalline and amorphous (quenched melt, QM) lactulose at $37^{\circ} \mathrm{C}$. Figure $7 \mathrm{a}$ corresponds to the amorphous lactulose (Xam $=1$ ). The heat flow curve shows a broad, but well defined, exothermic peak as expected for amorphous materials. The peak integration leads to a dissolution enthalpy close to $\Delta \mathrm{H}_{\text {dissol }}=57.4 \mathrm{~J} / \mathrm{g}$. This value is compatible with that determined by Miller et al. ${ }^{36}$ at $25^{\circ} \mathrm{C}(74 \mathrm{~J} / \mathrm{g})$. Indeed, Jasra ${ }^{43}$ shows that the exothermic contribution of the dissolution decreases when increasing the temperature. Figure $7 \mathrm{~b}$ corresponds to crystalline lactulose $(\mathrm{Xam}=0.0)$. It shows a quite sharp endotherm overlapping a broader exotherm. The resulting dissolution enthalpy determined by integration of the overall heat flow signal is $\Delta \mathrm{H}_{\text {dissol }}=-2.2 \mathrm{~J} / \mathrm{g}$, in agreement with the value obtained by Miller et al. ${ }^{36}$ at $25{ }^{\circ} \mathrm{C}(-7.11 \mathrm{~J} / \mathrm{g})$. This value is close to zero which means that the energy required to destroy the crystal lattice and that required to disperse the lactulose molecules in water, have the same order of magnitude. 
Moreover, the crystal destruction obviously precedes the dispersion lactulose molecules into water. The slight time shift between the two processes and their equivalent energy (while opposite) makes it possible the unusual detection of the two processes during the dissolution experiment. The strong difference between the dissolution enthalpies of crystalline and amorphous lactulose has been used to determine the amorphous fraction of lactulose samples regularly sampled during a two hour milling process. This has required the preliminary establishment of a calibration curve giving the dissolution enthalpy of the material as a function of its amorphous fraction. The details of the calibration are reported in the appendix (see supplementary data).

Heat flow curves recorded during the isothermal dissolution at $37{ }^{\circ} \mathrm{C}$ of crystalline lactulose samples having undergone milling operations of a duration varying from 3 min to $2 \mathrm{~h}$ can be found in supplementary data (see figure 9). The dissolution enthalpies of the milled crystalline lactulose samples, determined by integration of the dissolution peaks, are reported in supplementary data (see table 1). The fractions amorphized by milling (Xam) were then derived from the calibration law determined in the appendix (see supplementary data). These amorphous fractions are reported in supplementary data (see table 1) and their evolution with the milling time is illustrated in figure 8 . This evolution corresponds to a monotonous increase of the amorphous fraction. The experimental data could be correctly fitted by a simple exponential relaxation law of the type:

\section{$\operatorname{Xam}(t)=1-\exp (-t / \tau)$}

The best fit was obtained for a relaxation time $\tau=11 \mathrm{~min}$. This time is considerably shorter than the relaxation time usually observed in molecular materials for identical milling conditions. For example: chlorhexidine ${ }^{44}$, glucose ${ }^{7}$, lactose ${ }^{45}$, trehalose $^{46}$ and dexamethasone ${ }^{12}$ have relaxation times of about $1 \mathrm{~h}$ for identical milling conditions. Lactulose therefore appears to be a material that amorphizes particularly rapidly upon milling. Such a fast amorphization by milling might be due to weaker energy costs to create defects such as dislocations in lactulose compared to other sugars and thus to weaker elastic constants. To our knowledge, the elastic constants of lactulose have not yet been reported. To check the previous assumption, numerical elastic constants of lactulose and three other homologous disaccharides (sucrose, trehalose and lactose) were computed. The resulting elastic constants are reported in supplementary data (see table 2). As it can be noticed, elastic constants of lactulose material are smaller than those of other disaccharides. In particular, the lactulose shear modulus $\left(\mathrm{G}_{\mathrm{v}}=4.8 \mathrm{GPa}\right)$ is at least two times smaller than the shear modulus of other compounds (sucrose: $G_{v}=8.9 \mathrm{GPa}$; trehalose: $\mathrm{G}_{\mathrm{v}}=10.8$ $\mathrm{GPa}$; lactose: $\mathrm{G}_{\mathrm{v}}=10.5 \mathrm{GPa}$ ). This point could thus explain the faster amorphization of lactulose upon milling. 


\section{Conclusion}

In this paper, we have shown that crystalline lactulose can be amorphized, directly in the solid state, by mechanical milling. The amorphous lactulose thus obtained does not show any chemical degradation so that a reliable glass transition temperature of this material could be determined. This situation contrasts with the strong chemical degradation which occurs unavoidably during the conventional amorphization route of melt quenching. This degradation has been shown to have a strong anti-plasticization effect leading to strongly overestimated glass transition temperatures.

Interestingly, the amorphous state obtained by mechanical milling appears to be unusually stable and does not show any sign of recrystallization upon heating with moderate heating rates (typically $5^{\circ} \mathrm{C} / \mathrm{min}$ ). This stability is quite exceptional as most of milling induced amorphous materials recrystallize upon heating for similar heating conditions, even if the corresponding quenched liquids do not. We have taken advantage of this physical stability to procedure a nondegraded amorphous glass by the usual liquid quench technique. This was done by heating the milling induced amorphous lactulose just above $\mathrm{Tg}$ to get a metastable liquid which was then quenched well below Tg. The Tg of this glass was found to be identical to that of the milling induced amorphous material indicating that the alpha relaxation processes involved in the manifestation of the glass transition could be independent of the amorphization route.

The amorphization kinetic of lactulose upon milling has been determined in an original way by isothermal dissolution calorimetry. It is shown here that this technique is effective to determine the amorphous content of a material when conventional characterization techniques, like DSC and powder X-diffraction, can hardly be used. The amorphization kinetics of lactulose has been found to be extremely fast compared to similar materials and to obey an exponential relaxation law. These last two characteristics are consistent as an exponential relaxation law is expected for systems where each impact readily amorphizes the corresponding impacted fraction of the material ${ }^{47}$.

\section{Acknowledgment}

One of the authors (F.N.) is grateful to ILL (Institute Laue Langevin) and the University of Lille for their financial support. This project has received funding from the Interreg 2 Seas programme 2014-2020 co-funded by the European Regional Development Fund under subsidy contract 2S01-059_IMODE. 


\section{CAPTIONS FIGURES}

Figure 1: Powder X-ray diffraction patterns of lactulose recorded at RT. From bottom to top: crystalline form (black line), quenched melt (QM, green line), crystal milled $8 \mathrm{~h}$ (MIL, red line).

Figure 2: DSC scans of lactulose recorded at $5{ }^{\circ} \mathrm{C} / \mathrm{min}$ :

run 1 , heating of the crystalline form

run 2 , cooling of the melt

run 3 , heating of the quenched melt.

Figure 3: a) TGA scan (black line) and derivative TGA scan (blue line) of crystalline lactulose recorded after $8 \mathrm{~h}$ of milling $\left(5^{\circ} \mathrm{C} / \mathrm{min}\right)$.

b) Heating DSC scans $\left(5^{\circ} \mathrm{C} / \mathrm{min}\right)$ of lactulose:

Run 1: $8 \mathrm{~h}$ milled crystalline form - total (red solid line) and reversible (red dashed line) signals

Run 2: quenched melt (green line).

Figure 4: a) Repeated DSC scans $\left(5^{\circ} \mathrm{C} / \mathrm{min}\right)$ of crystalline lactulose milled $8 \mathrm{~h}$. For each run, the end temperature is increased by $10^{\circ} \mathrm{C}$ compared to the previous one. At the end of each run the sample has been rapidly cooled to $20^{\circ} \mathrm{C}$.

b) Schematic illustration of the thermal treatment used to perform the DSC scan from run 1 to run 7 .

Figure 5: XRD patterns of anhydrous crystalline lactulose recorded after different milling times ranging from 0 to $8 \mathrm{~h}$. The XRD pattern of the QM is reported for comparison. All patterns were recorded at room temperature.

Figure 6: Heating DSC curves $\left(5^{\circ} \mathrm{C} / \mathrm{min}\right)$ of crystalline lactulose samples recorded after different milling times ranging from 0 to $8 \mathrm{~h}$. The DSC curve of the QM is reported for comparison.

Figure 7: Heat flow curves recorded during the isothermal dissolution at $37^{\circ} \mathrm{C}$ of crystalline (7 a) and QM (7 b) lactulose samples.

Figure 8: Amorphization kinetic of crystalline lactulose under milling. The green line is the best fit of the data with an exponential law. Error bars are the mean squared error 
of the dissolution enthalpy obtained after three measurements on the crystalline lactulose. 
1. Willart JF, Descamps M 2008. Solid State Amorphization of Pharmaceuticals. Molecular Pharmaceutics 5(6):905-920.(10.1021/mp800092t)

2. Brittain HG 2002. Effects of mechanical processing on phase composition. Journal of Pharmaceutical Sciences 91(7):1573-1580.(10.1002/jps.10115)

3. Tsukushi I, Yamamuro O, Matsuo T 1995. Solid state amorphization of organic molecular crystals using a vibrating mill. Solid State Communications 94(12):1013-1018

4. Descamps M, Willart JF 2016. Perspectives on the amorphisation/milling relationship in pharmaceutical materials. Advanced Drug Delivery Reviews 100:5166.(http://dx.doi.org/10.1016/i.addr.2016.01.011)

5. De Gusseme A, Neves C, Willart JF, Rameau A, Descamps M 2008. Ordering and disordering of molecular solids upon mechanical milling: The case of fananserine. Journal of Pharmaceutical Sciences 97(11):5000-5012.(10.1002/jps.21472)

6. Descamps M, Willart JF, Dudognon E, Caron V 2007. Transformation of pharmaceutical compounds upon milling and comilling: The role ofTg. Journal of Pharmaceutical Sciences 96(5):13981407.(10.1002/jps.20939)

7. Dujardin N, Willart J, Dudognon E, Hedoux A, Guinet $Y$, Paccou L, Chazallon B, Descamps M 2008. Solid state vitrification of crystalline $\alpha$ and $\beta$-D-glucose by mechanical milling. Solid State Communications 148(1-2):78-82.(10.1016/j.ssc.2008.07.002)

8. Crowley KJ, Zografi G 2002. Cryogenic grinding of indomethacin polymorphs and solvates: Assessment of amorphous phase formation and amorphous phase physical stability. Journal of Pharmaceutical Sciences 91(2):492-507

9. Qi S, Weuts I, De Cort S, Stokbroekx S, Leemans R, Reading M, Belton P, Craig DQM 2010. An investigation into the crystallisation behaviour of an amorphous cryomilled pharmaceutical material above and below the glass transition temperature. Journal of Pharmaceutical Sciences 99(1):196208.(10.1002/jps.21811)

10. Megarry AJ, Booth J, Burley J 2011. Amorphous trehalose dihydrate by cryogenic milling. Carbohydrate Research.(10.1016/j.carres.2011.03.011)

11. Einfalt T, Planinsek O, Hrovat K 2013. Methods of amorphization and investigation of the amorphous state. Acta Pharm 63:305-334

12. Oliveira PFM, Willart J-F, Siepmann J, Siepmann F, Descamps M 2018. Using Milling To Explore Physical States: The Amorphous and Polymorphic Forms of Dexamethasone. Crystal Growth \& Design 18(3):1748-1757.(10.1021/acs.cgd.7b01664)

13. Craig DQM, Royall PG, Kett VL, Hopton ML 1999. The relevance of the amorphous state to pharmaceutical dosage forms: glassy drugs and freeze dried systems. International-journal-ofpharmaceutics 179(2):179-207.(10.1016/s0378-5173(98)00338-x)

14. Haque MK, Roos YH 2005. Crystallization and X-ray diffraction of spray-dried and freeze-dried amorphous lactose. Carbohydrate Research 340(2):293-301.(10.1016/j.carres.2004.11.026)

15. Schammé B, Couvrat N, Malpeli P, Dudognon E, Delbreilh L, Dupray V, Dargent É, Coquerel G 2016. Transformation of an active pharmaceutical ingredient upon high-energy milling: A processinduced disorder in Biclotymol. International-journal-of-pharmaceutics 499(1):6773.(https://doi.org/10.1016/ji.ijpharm.2015.12.032)

16. Willart JF, Durand M, Briggner LE, Marx A, Danède F, Descamps M 2013. Solid-state amorphization of linaprazan by mechanical milling and evidence of polymorphism. Journal of Pharmaceutical Sciences 102(7):2214-2220.(10.1002/jps.23573)

17. Adrjanowicz K, Kaminski K, Grzybowska K, Hawelek L, Paluch M, Gruszka I, Zakowiecki D, Sawicki W, Lepek P, Kamysz W, Guzik L 2011. Effect of Cryogrinding on Chemical Stability of the Sparingly Water-Soluble Drug Furosemide. Pharmaceutical Research 28(12):32203236.(10.1007/s11095-011-0496-4)

18. Trasi NS, Byrn SR 2012. Mechanically Induced Amorphization of Drugs: A Study of the Thermal Behavior of Cryomilled Compounds. AAPS PharmSciTech 13(3):772-784.(10.1208/s12249-012-98018) 
19. Sussich F, Cesàro A 2008. Trehalose amorphization and recrystallization. Carbohydrate Research 343(15):2667-2674.(10.1016/j.carres.2008.08.008)

20. Chattoraj S, Bhugra C, Telang C, Zhong L, Wang Z, Sun CC 2011. Origin of Two Modes of Nonisothermal Crystallization of Glasses Produced by Milling. Pharmaceutical Research (29):113.(10.1007/s11095-011-0644-x)

21. Yu L 2016. Surface mobility of molecular glasses and its importance in physical stability. Advanced Drug Delivery Reviews 100:3-9.(10.1016/j.addr.2016.01.005)

22. Wu T, Yu L 2006. Surface crystallization of indomethacin below T g. Pharmaceutical Research 23(10):2350-2355.(10.1007/s11095-006-9023-4)

23. Zhu L, Wong L, Yu L 2008. Surface-Enhanced Crystallization of Amorphous Nifedipine. Molecular Pharmaceutics 5(6):921-926.(10.1021/mp8000638)

24. Shi Q, Cai T 2016. Fast Crystal Growth of Amorphous Griseofulvin: Relations between Bulk and Surface Growth Modes. Crystal Growth and Design 16(6):3279-3286.(10.1021/acs.cgd.6b00252)

25. Mizota T, Suzawa I, Seki N, Tamura Y, Shimamura S 1994. Solubility of lactulose trihydrate. Carbohydrate Research 263(1):163-166.(10.1016/0008-6215(94)00154-5)

26. Smith W, Forester TR, Todorov IT 2012. The DL_POLY Classic User Manual, 1.9. STFC Daresbury Laboratory

27. Damm W, Frontera A, Tirado-Rives J, Jorgensen WL 1997. OPLS all-atom force field for carbohydrates. Journal of Computational Chemistry 18(16):1955-1970.(10.1002/(sici)1096987x(199712)18:16<1955::aid-jcc1>3.3.co;2-a)

28. Kony D, Damm W, Stoll S, Van Gunsteren WF 2002. An improved OPLS-AA force field for carbohydrates. Journal of Computational Chemistry 23(15):1416-1429.(10.1002/jcc.10139)

29. Clavier G, Desbiens N, Bourasseau E, Lachet V, Brusselle-Dupend N, Rousseau B 2017. Computation of elastic constants of solids using molecular simulation: comparison of constant volume and constant pressure ensemble methods. Molecular Simulation 43(17):14131422.(10.1080/08927022.2017.1313418)

30. Jeffrey GA, Wood RA, Pfeffer PE, Hicks KB 1983. Crystal structure and solid-state NMR analysis of lactulose. Journal of the American Chemical Society 105(8):2128-2133

31. Hanson JC, Sieker LC, Jensen LH 1973. Sucrose: X-ray refinement and comparison with neutron refinement. Acta Crystallographica Section B Structural Crystallography and Crystal Chemistry 29(4):797-808.(10.1107/s0567740873003365)

32. Jeffrey GA, Nanni R 1985 . The crystal structure of anhydrous $\alpha, \alpha$-trehalose at $-150^{\circ}$. Carbohydrate Research 137:21-30.(10.1016/0008-6215(85)85146-6)

33. Platteau C, Lefebvre J, Affouard F, Willart J-F, Derollez P, Mallet F 2005. Structure determination of the stable anhydrous phase of $\alpha$-lactose from $\mathrm{X}$-ray powder diffraction. Acta Crystallographica Section B Structural Science 61(2):185-191.(10.1107/s0108768105000455)

34. Zhou Y-C, Zhao C, Wang F, Sun Y-J, Zheng L-Y, Wang X-H 2013. Theoretical Prediction and Experimental Investigation on the Thermal and Mechanical Properties of Bulk $\beta-Y b<$ sub $>2</ s u b>\mathrm{Si}$ $<$ sub $>2</$ sub $>0<$ sub $>7</$ sub $>$. Journal of the American Ceramic Society 96(12):38913900.(10.1111/jace.12618)

35. Rodriguez-Carvajal J. 2017. Crystallographic tools for Rietveld, profile matching and integrated intensity refinements of X-Ray and/or neutron data. ed.:

36. Miller DP, de Pablo JJ 2000. Calorimetric solution properties of simple saccharides and their significance for the stabilization of biological structure and function. Journal of Physical Chemistry $B$ 104(37):8876-8883.(Doi 10.1021/Jp000807d)

37. Salah AM. 2015. Effets du broyage mécanique sur l'état physique des matériaux pharmaceutiques vitreux. ed.:

38. Bustin O, Descamps M 1999. Slow structural relaxations of glass-forming Maltitol by modulated DSC calorimetry. The Journal of Chemical Physics 110(22):1098210992.(10.1063/1.478041)

39. Chen HS, Wang TT 1981. Sub-sub Tg structural relaxation in glassy polymers. Journal of Applied Physics 52(10):5898-5902.(10.1063/1.329827) 
40. Hodge IM 1994. Enthalpy relaxation and recovery in amorphous materials. Journal of NonCrystalline Solids 169(3):211-266.(10.1016/0022-3093(94)90321-2)

41. Descamps M, Aumelas A, Desprez S, Willart JF 2015. The amorphous state of pharmaceuticals obtained or transformed by milling: Sub-Tg features and rejuvenation. Journal of Non-Crystalline Solids 407:72-80.(10.1016/j.jnoncrysol.2014.08.055)

42. Jackson CL, McKenna GB 1990. The melting behavior of organic materials confined in porous solids. The Journal of Chemical Physics 93(12):9002.(10.1063/1.459240)

43. Jasra RV, Ahluwalia JC 1984. Enthalpies and heat capacities of dissolution, apparent molar heat capacities, and apparent molar volumes of some mono-, di-, tri-, and tetra-saccharides in water. The Journal of Chemical Thermodynamics 16(6):583-590.(10.1016/0021-9614(84)90010-7)

44. Elisei E, Willart J-F, Danède F, Siepmann J, Siepmann F, Descamps M 2017. Crystalline Polymorphism Emerging From a Milling-Induced Amorphous Form: The Case of Chlorhexidine Dihydrochloride. Journal of Pharmaceutical Sciences.(10.1016/j.xphs.2017.07.003)

45. Caron V, Willart JF, Lefort R, Derollez P, Danède F, Descamps M 2011. Solid state amorphization kinetic of alpha lactose upon mechanical milling. Carbohydrate Research 346(16):26222628.(10.1016/j.carres.2011.09.004)

46. Bordet P, Bytchkov A, Descamps M, Dudognon E, Elkaïm E, Martinetto P, Pagnoux W, Poulain A, Willart JF 2016. Solid State Amorphization of $\beta$-Trehalose: A Structural Investigation Using Synchrotron Powder Diffraction and PDF Analysis. Crystal Growth and Design 16(8):45474558.(10.1021/acs.cgd.6b00660)

47. Delogu F, Cocco G 2000. Relating Single-Impact Events to Macrokinetic Features in Mechanical Alloying Processes. Journal of Materials Synthesis and Processing 8(5):271277.(10.1023/a:1011382008963) 
Figure 1

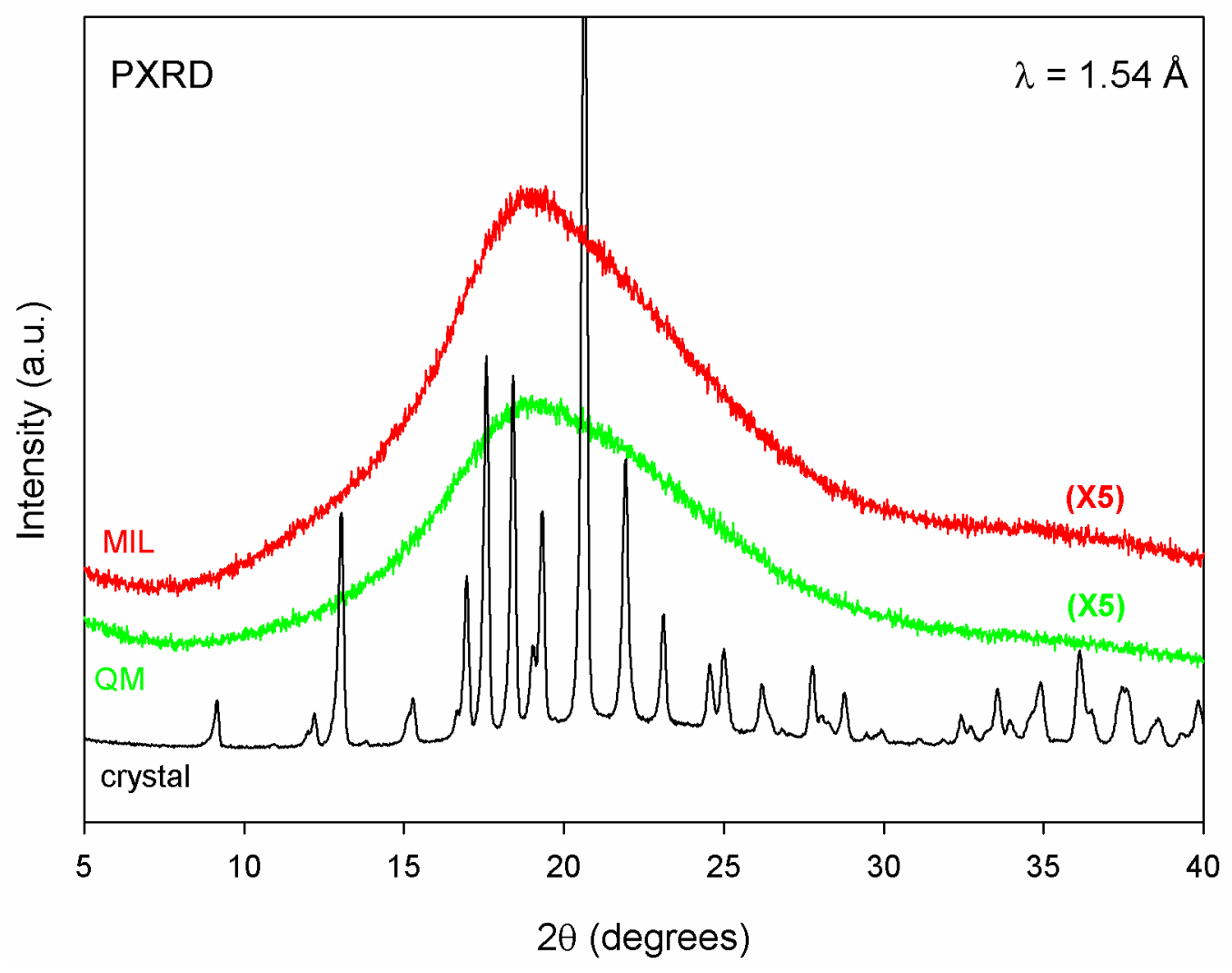


Figure 2

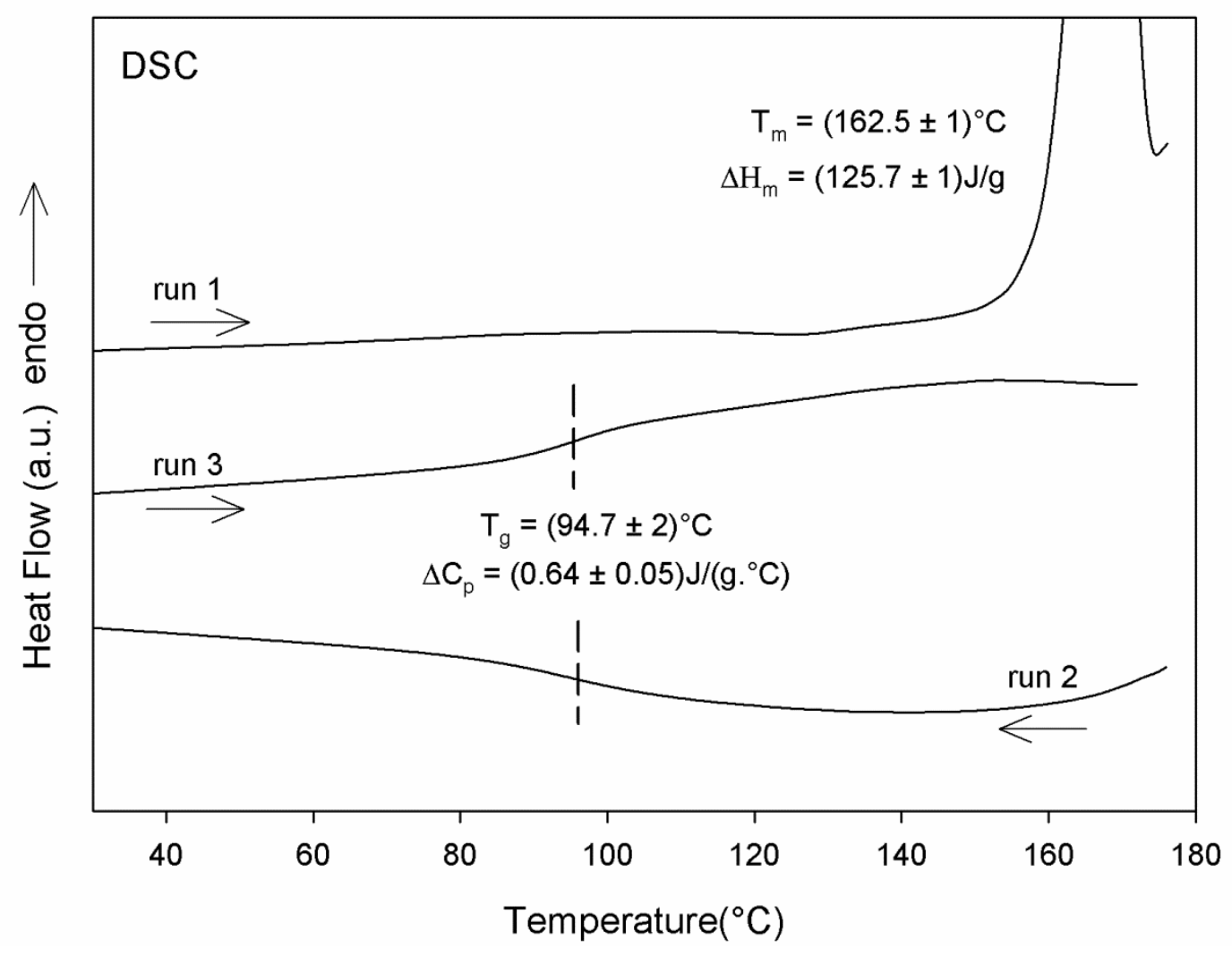


Figure 3

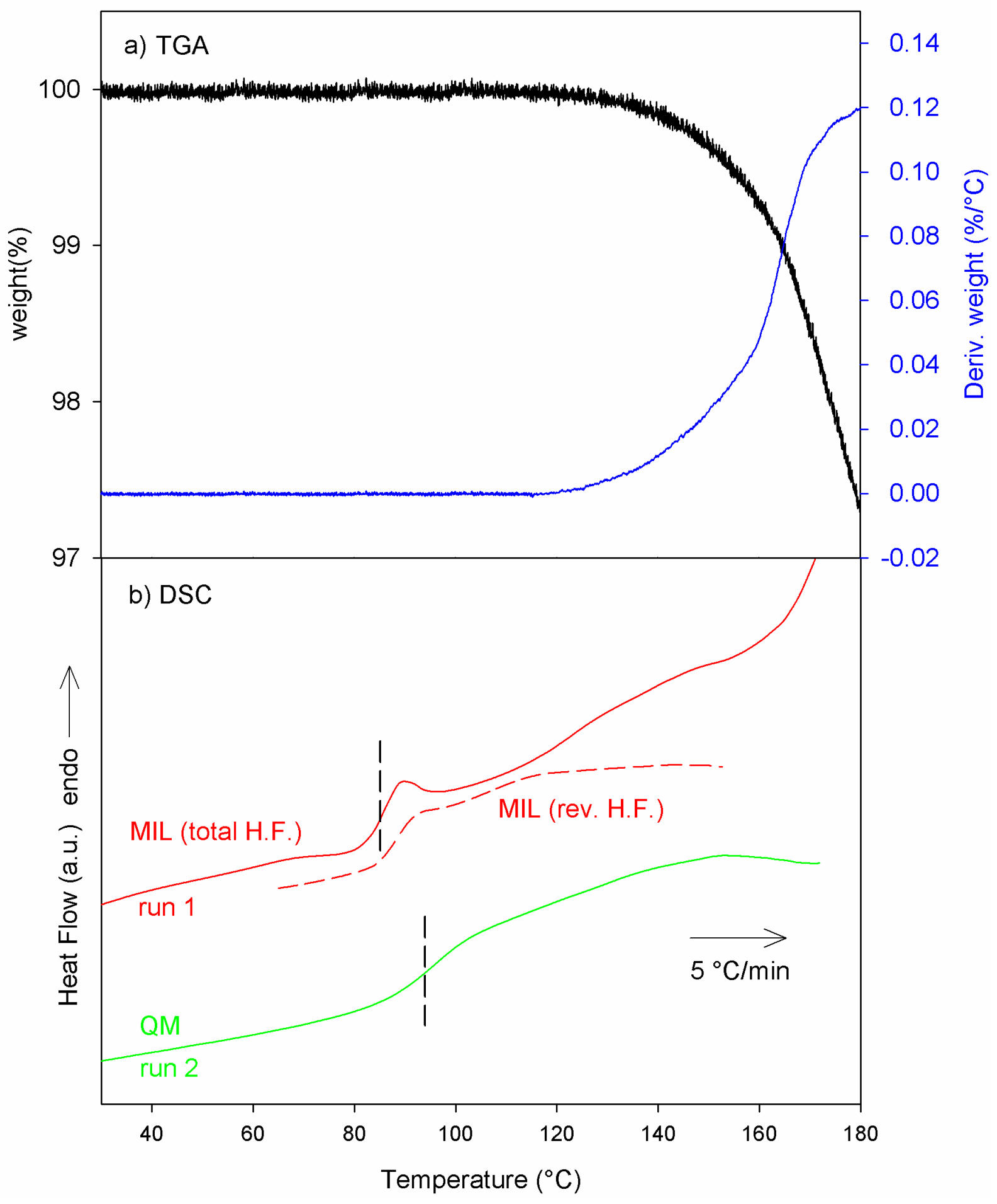


Figure 4
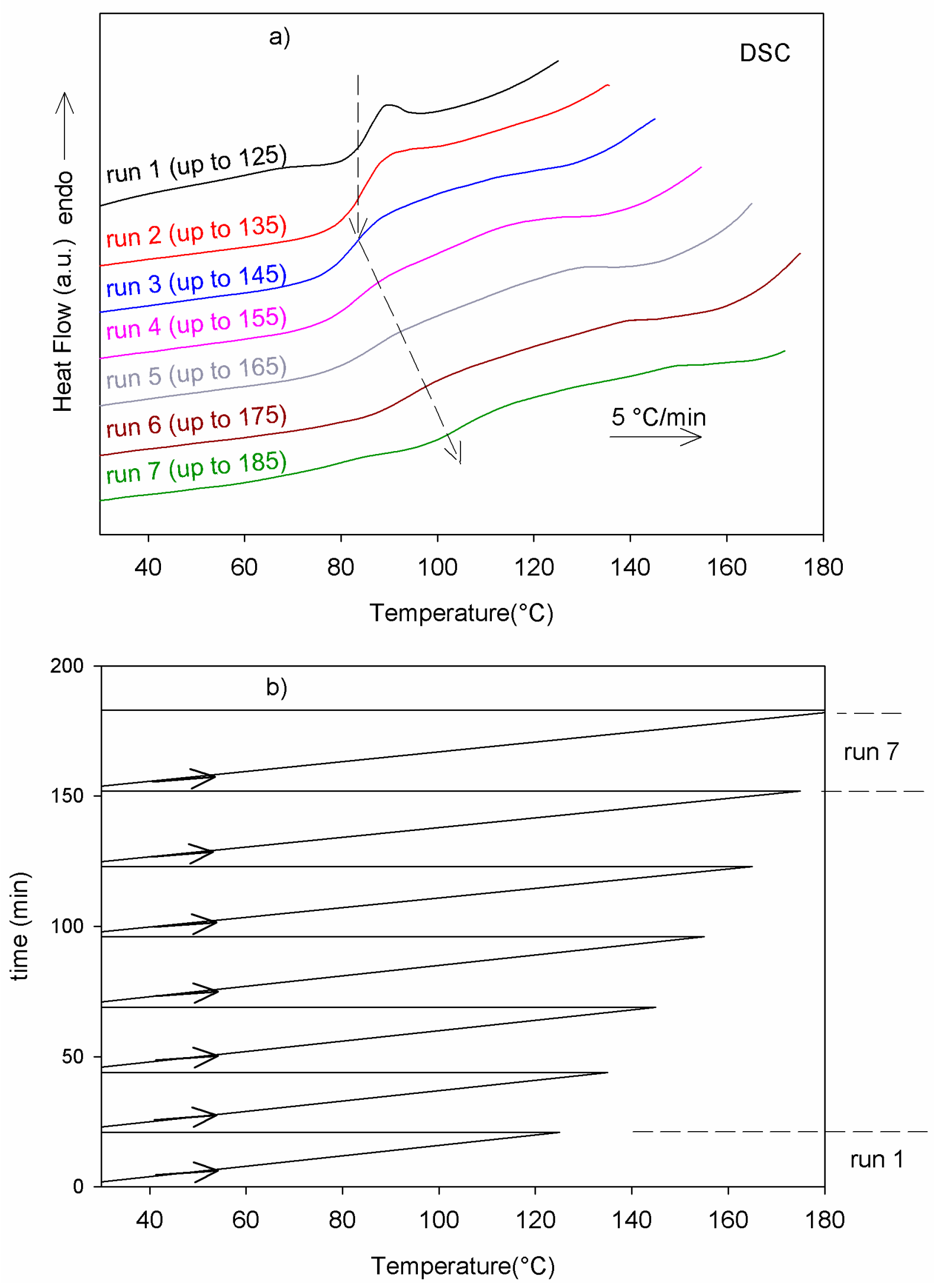
Figure 5

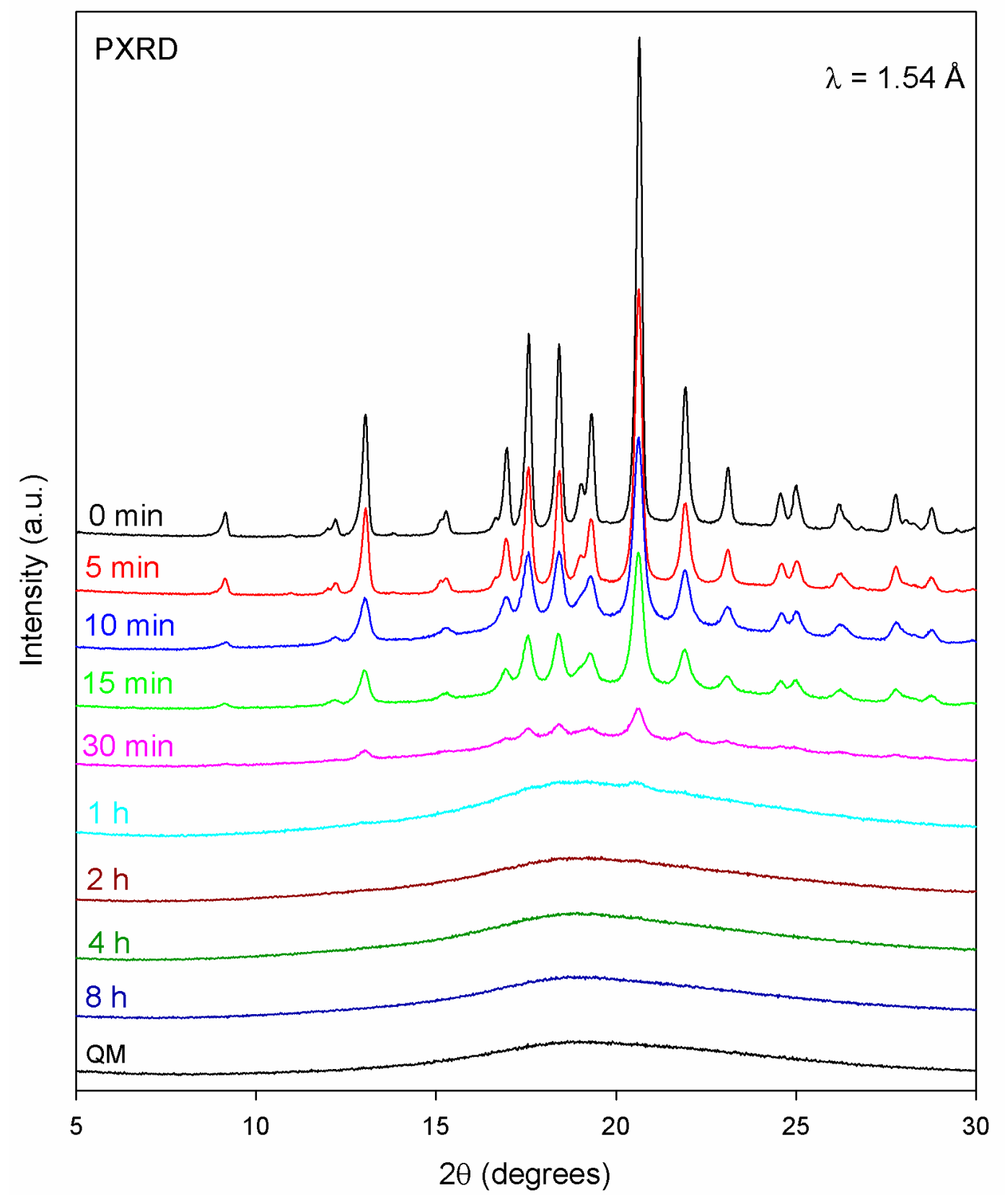


Figure 6

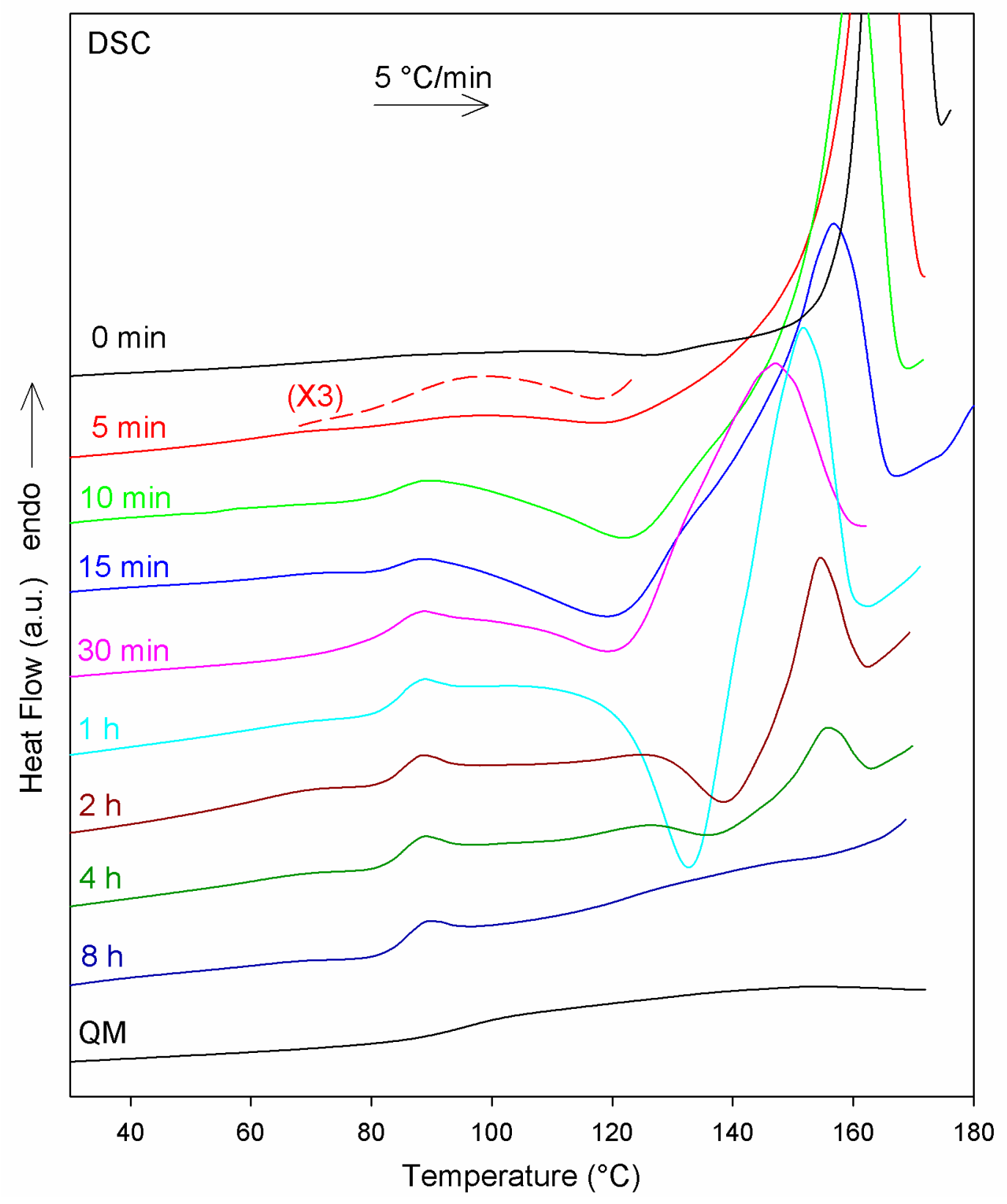


Figure 7
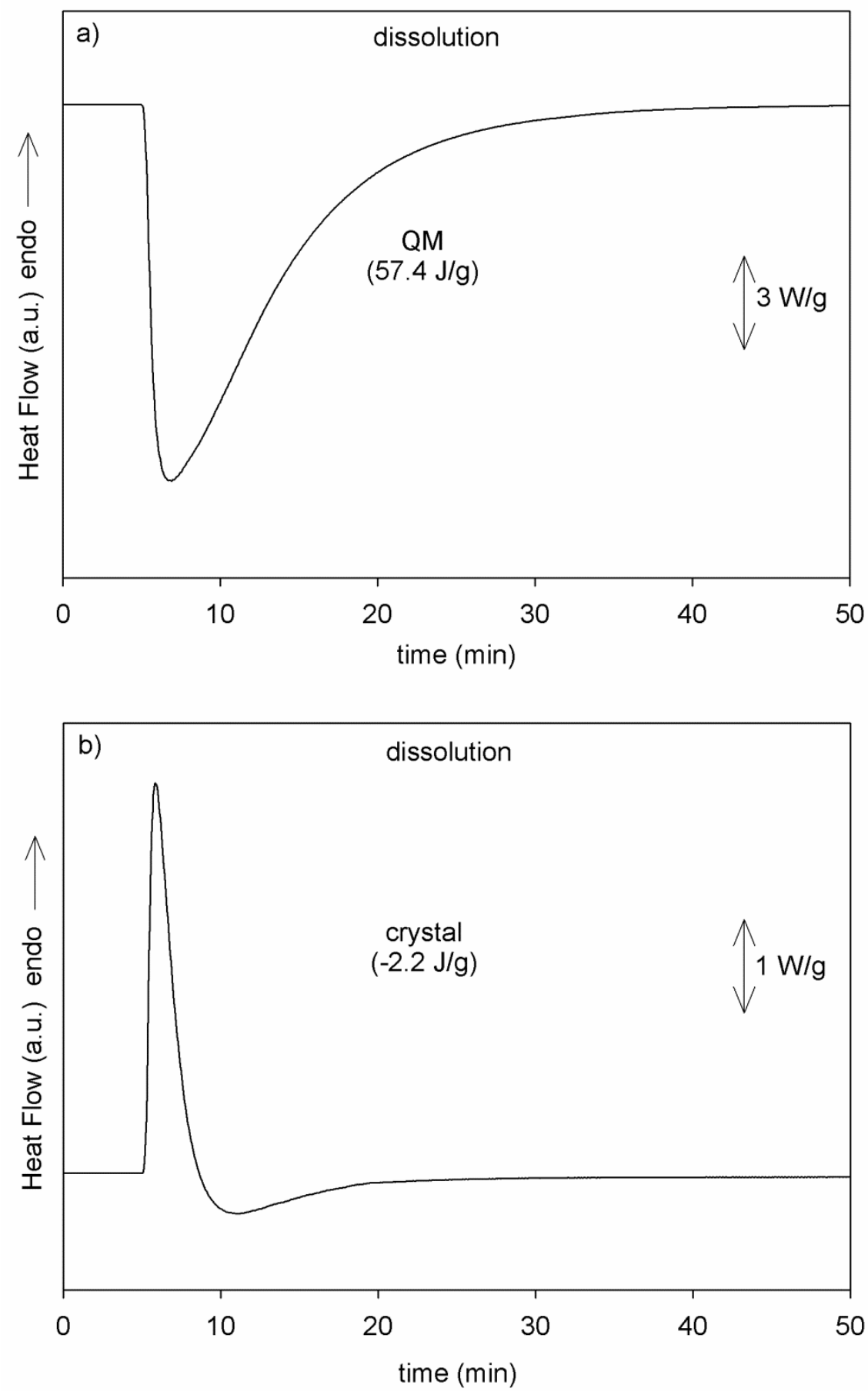
Figure 8

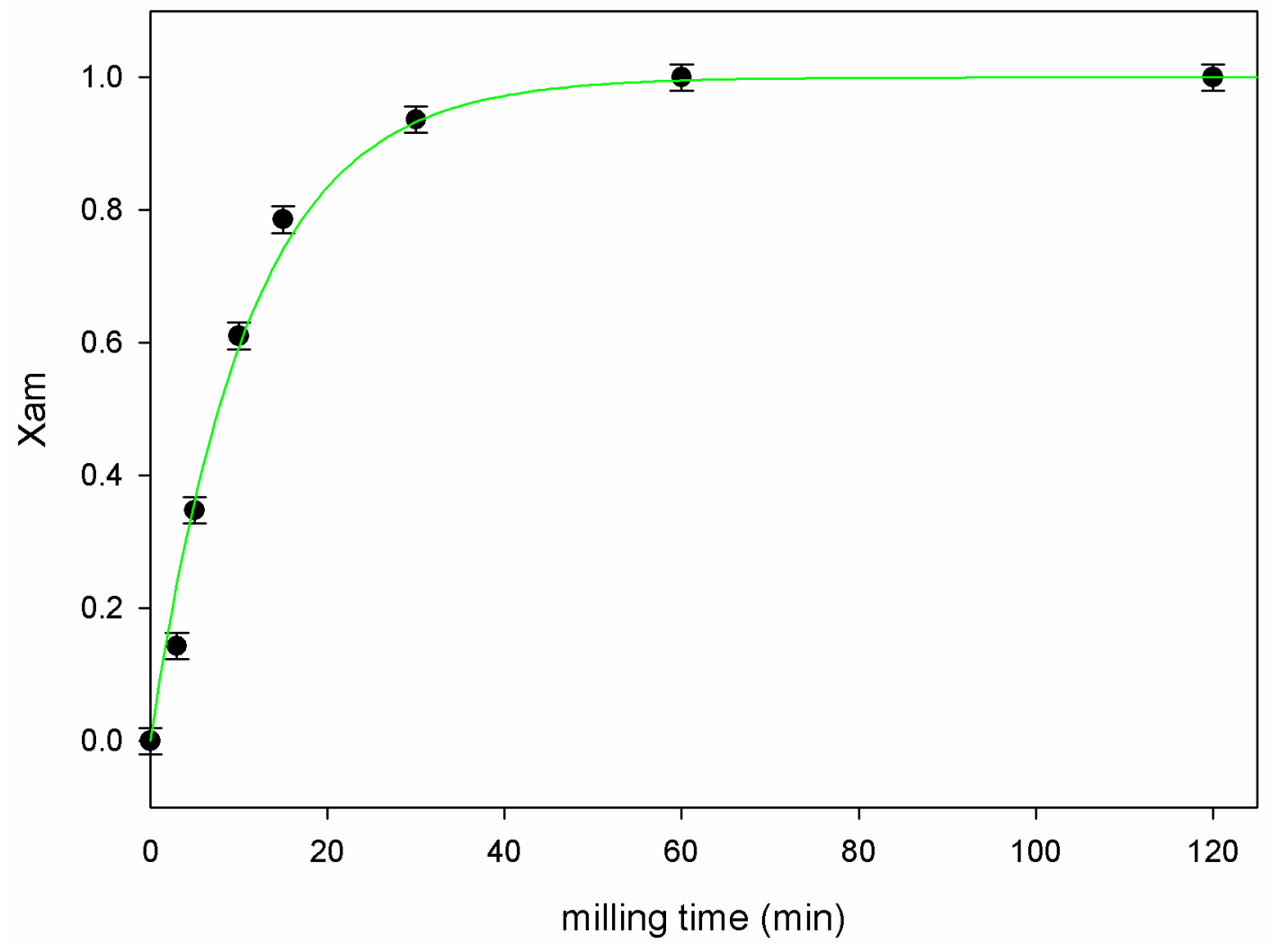

